\title{
Influence of Knowledge about Cardio-Cerebrovascular Disease Symptoms on Self-Management Behaviors in Patients with Atrial Fibrillation
}

\author{
YunHee Oh$^{1}$, Sun Yi Yang ${ }^{2}$ \\ ${ }^{1}$ Assistant Professor, Department of Nursing, Cheju Halla University Jeju-do, Republic of Korea 38, Halladaehak- \\ ro, Jeju-si, Jeju-do, 63092, Korea, ${ }^{2}$ Assistant Professor, College of Nursing, Daejeon Medical Campus, Konyang \\ University, Daejeon, Republic of Korea 158, Gwanjeodong-ro, Seo-gu, Daejeon 35365, Korea
}

\begin{abstract}
Aim: This study aimed to assess stroke risk and Cardio-cerebrovascular symptom knowledge in patients with atrial fibrillation, and to determine the relationship between knowledge and self-management in these patients.

Methods: Using a cross-sectional study design, 120 patients from an outpatient clinic were recruited from two medical centers. Data were collected using a structured questionnaire, and data on risk factors and clinical characteristics were collected from patients' medical records. The data were analyzed by one-way analysis of variance, Pearson correlation coefficient, and hierarchical multiple linear regression analysis.

Results: The risk of stroke was estimated to be $82.5 \%$. For the assessment of patient cardio-cerebrovascular symptom knowledge the correct answer rate was as $37 \%$. Hierarchical regression analysis showed that knowledge about cardio-cerebrovascular disease symptoms was a significant predictor, explaining an additional $28.3 \%$ of the variance of self-management behaviors.

Conclusions: These results suggest the need for education programs that include information about cardiocerebrovascular disease symptoms and the risks of stroke occurring as a complication, in order to enhance self-management behaviors in patients with atrial fibrillation.
\end{abstract}

Key words: Atrial fibrillation, Cardiovascular disease, Cerebrovascular disease, Knowledge, Selfmanagement

\section{Introduction}

In atrial fibrillation (AF), the most common arrhythmia, electrical flow occurs in various parts of the atrium, causing irregular and rapid tremors of heart contraction $^{[1]}$. In a study of 1,483 elderly people over 60 years of age, atrial fibrillation was diagnosed by

\section{Corresponding author: \\ YunHee Oh,}

Assistant Professor, Department of Nursing, Cheju Halla University, Jeju-do, Republic of Korea 38, Halladaehak-ro, Jeju-si, Jeju-do, 63092, Korea Tel: +82-064-741-6703 Fax: +82-64-741-7639 e- mail: yunheeoh2020@gmail.com electrocardiogram screening in $1.0 \%$ of participants in their $60 \mathrm{~s}, 3.3 \%$ in their $70 \mathrm{~s}$, and $7.2 \%$ in their $80 \mathrm{~s}^{[2]}$. The prevalence rate is expected to increase with the aging population ${ }^{2}$. AF presents symptoms such as palpitations, dizziness, fainting, and chest pain, and increases the risk of heart failure with reduced ventricular function and stroke due to blood clots formed by the accumulation of blood ${ }^{[3]}$. Complication morbidity doubles mortality in women and increases mortality in men by 1.5 fold $^{[3]}$, increases risk of stroke by 5 times, risk of heart failure by 3 times, and dementia by 2 times $^{[1]}$.

In one stroke registration study, $19 \%$ of ischemic cerebral infarction was found to be caused by $\mathrm{AF}^{[4]}$. $\mathrm{AF}$ was reported to have high severity and mortality ${ }^{[5]}$. In 
most patients with AF, their condition is associated with hypertension, diabetes, and hyperlipidemia, which are risk factors for cardio-cerebrovascular disease(CVD ${ }^{[6]}$. According to AF management guidelines, antithrombotic treatment should be determined by estimating the stroke risk of AF patients to prevent the occurrence of $\mathrm{CVD}^{[1]}$. Because the choice of AF treatment depends on symptoms and stroke risk, it is necessary to first understand the stroke risk level of AF patients in order to effectively manage AF.

One study found that most patients with AF were unaware of their risk for CVD. In that study, the highrisk group showed no difference in stroke perception compared with the low-risk group ${ }^{[7]}$. In order to prevent delays in the treatment of stroke, raising awareness of the symptoms is essential. The American Heart Association privides AF patient education that includes stroke risk categories and stroke symptoms ${ }^{[8]}$. AF guidelines suggest that appropriate treatment to prevent aggravation of the disease includes awareness of possible heart failure ${ }^{[1]}$. Therefore, patients with $\mathrm{AF}$, a disease that can progress to fatal CVD, should be assessed and provided with adequate education on the symptoms related to the expected complications. Self-management in patients with $\mathrm{AF}$ requires continuous medication and lifestyle management to prevent disease exacerbation and to prevent complications ${ }^{[3]}$. Self-management behavior strategies include preventive health behaviors, such as perception of disease that recognizes symptoms and risk factors, drug side effects management, pulse monitoring for symptom monitoring, and awareness of complications $^{[9]}$. Patients with AF need to understand the importance of early detection and coping with CVD in order to improve their lifestyle and prevent complications by adjusting to long-term treatment. Therefore, it is necessary to confirm whether knowledge about symptoms affects the self-management behavior of patients with $\mathrm{AF}$.

\section{Aims}

The purpose of this study was to assess the risk of stroke, CVD symptom knowledge, and self-management behavior, and to identify the factors that affect selfmanagement behavior in patients with $\mathrm{AF}$.
- Determine participant stroke risk, CVD symptom knowledge, and self-management behavior.

- Explore differences in CVD symptom knowledge and self-care behavior according to the general characteristics of the participants and the risk of stroke.

- Identify the effect of patient CVD symptom knowledge on self-management behavior.

\section{Method}

\section{Study design and participats}

This study used a descriptive research design. The study participants were patients with AF who visited outpatient cardiac units at two general hospitals. The inclusion criteria were official diagnoses of $\mathrm{AF}$ and $\mathrm{AF}$ for more than 3 months. The exclusion criteria were having been diagnosed less than 3 months prior to the study and changed treatment within the past 3 months. Using the $\mathrm{G}^{*}$ Power 3.1 program, the number of participants was determined based on the calculations of previous studies $^{[10]}$, with a median effect size of 15 , regression analysis with a significance level of 05 , a power of $80 \%$, and 8 independent variables (age, gender, education level, family structure, economic status, duration of illness, stroke risk, and knowledge about symptoms). Considering a dropout rate of $10 \%$, it was determined 125 initial participants were required to ensure a total of at least 109 participants. Data were collected on 123 patients. There were 3 incomplete questionnaires, and therefore the data from 120 participants were analyzed.

\section{Measures}

\section{Stroke risk}

The $\mathrm{CHA}_{2} \mathrm{DS}_{2}$-VASc score (congestive heart failure, hypertension, age $\geq 75$ [doubled], diabetes mellitus, prior stroke or transient ischemic attack [doubled], vascular disease, age 65-74, female) was used to assess the risk of stroke ${ }^{[11]}$. In the calculation of the CHA $\square \mathrm{DS} \square$-VASc score, 1 point is added to the score for the presence of congestive heart failure, hypertension, diabetes, or vascular disease, 2 points for being female, 2 points for having a history of stroke, 2 points for being over 75 years of age, and 1 point for being between 65 and 74 years of age. A combined score of 0 indicates low risk, 1 
indicates medium risk, and 2 or more indicates high risk.

Cardio-cerebrovascular(CVD)

\section{Knowledge}

symptom

CVD symptom knowledge was measured by the American Heart Association's revised instrument for warning signs of stroke, myocardial infarction, and heart failure ${ }^{[12]}$. Items of the instrument are on five stroke symptoms, five myocardial infarction symptoms, and seven heart failure symptoms. In the scoring of the measurement, a correct answer is given 1 point. "Do not know" or incorrect answers are given 0 points. The total score ranges from 0 to 17 points. Higher scores indicate more CVD symptom knowledge. For this instrument, the Kuder-Richardson 20 in study ${ }^{[12]}$ and in the current study were .74 and .91 , respectively.

\section{Self-management behaviors}

A questionnaire developed by $\mathrm{Xu}$ et $\mathrm{al}^{[13]}$. was used to measure self-management behaviors of patients with AF. Permission to translate the questionnaire into Korean was obtained from the original authors. The questionnaire was back-translated by a bilingual person proficient in both Korean and English. The Korean version was validated using content validity. The original questionnaire consisted of 13 items: 4 items on adherence to medication regimen, international normalized ratio monitoring, periodic follow-up, and daily pulse self-examination, and 6 on healthy lifestyle. The questionnaire items were measured on a 4-point Likert scale that ranged from 1 ("neither agree nor disagree") to 4 ("strongly agree"); the scores ranged from 13 to 52, with higher scores indicating greater selfmanagement behaviors. Cronbach's $\alpha$ in Xu et al ${ }^{[13]}$. and in the current study were .86 and .79 , respectively.

\section{Data Collection}

Data collection was carried out from March 2017 to September 2017 at the two outpatient cardiac units. Consent was obtained from the medical teams and medical staff. The questionnaire was self-administered, and the researcher and research assistant provided assistance when necessary. The questionnaire took about 20-30 minutes to complete. The terms of AF prevalence and stroke risk were checked with the electronic medical records.

\section{Ethical Consideration}

This study was conducted after approval from the research ethics committee of the institution (IRB No. 2017-02-007-004) collecting the data. The researcher explained the purpose, methods, and anonymity of the study to the study participants and informed them that taking part in the study was voluntary. Each participant provided prior written informed consent.

\section{Data Analysis}

Data were analyzed using IBM SPSS 22.0. Instrument reliability was assessed using Cronbach's $\alpha$. Data on general characteristics, disease-related characteristics, stroke risk, CVD symptom knowledge, and self-management behavior were analyzed via real numbers, percentage, average, and standard deviation. Self-management behaviors was verified by Kolmogorov-Smirnov test ( score $=.065, \mathrm{p}=.120$ ), and the assumption of equal variance was confirmed by Levene's statistics. Differences in CVD symptom knowledge and self-management behaviors were determined using a one-way analysis of variance, and post-hoc testing was performed with the Scheffé test. The correlation between CVD symptom knowledge and self-management behavior was examined using Pearson's correlation coefficients, and a hierarchical regression analysis was performed to determine the effect on self-management behavior. In the testing of the regression model for multicollinearity, it was found that the correlation was $.03 \sim .59$, less than 0.8 , the tolerance was $0.98,0.1$ or more, and the variation inflation factor was 1.37 , less than 10. The Durbin-Watson statistic was 2.39 , close to 2 . There was no problem of autocorrelation.

\section{Results}

\section{Demographic and disease-related characteristics of the participants}

The average age of the participants was $69.3 \pm$ 11.5 years, and 71 were males (59.2\%). With regard to education level, $42.5 \%$ had elementary school education and under, and $85.0 \%$ lived with their families. The mean duration of AF was $46.2 \pm 39.1$ months. The major 
comorbidities were hypertension (56.7\%), diabetes (25.8\%), and peripheral vascular disease (17.5\%). Among the medication used by the participants, 37.5\% were new oral anticoagulants; $33.3 \%$ of the patients used warfarin. The risk of stroke, assessed by the $\mathrm{CHA}_{2} \mathrm{DS}_{2}-$ VASc score, was $7.5 \%$ in the low-risk group, $10.0 \%$ in the middle-risk group, and $82.5 \%$ in the high-risk group. The most common risk factor for stroke, as determined by the $\mathrm{CHA}_{2} \mathrm{DS}_{2}$-VASc score, was an age of 65 years or over $(66.7 \%)$, followed by hypertension $(56.7 \%)$, being female (40.8\%), and having diabetes (25.8\%) (Table 1).

\section{CVD symptom knowledge and self-management behavior}

The average score for CVD symptom knowledge was $6.30 \pm 5.12$ points, out of a possible 17 points, with a correct answer rate of $44 \%$ for stroke, $34 \%$ for myocardial infarction, and $32 \%$ for heart failure. The average score for self-management behavior was $35.32 \pm 6.51$, out of a possible 52 points (Table 2 ). The correlation between CVD symptom knowledge and self-management behavior was examined by univariate analysis $(\mathrm{r}=.57, \mathrm{p}<.001)$.

Differences in CVD symptom knowledge and self-management behaviors according to participant

\section{characteristics and stroke risk}

There were statistically significant differences in CVD symptom knowledge according to age $(\mathrm{F}=4.51$, $\mathrm{p}=.012)$ and education level $(\mathrm{F}=6.88 \mathrm{p}<.001)$. Selfmanagement behaviors were significantly different according to age $(\mathrm{F}=6.49 \mathrm{p}=.002)$, education level $(\mathrm{F}=6.29 \mathrm{p}=.001)$, and duration of disease $(\mathrm{F}=3.97 \mathrm{p}$ $=.021)$. However, there was no statistically significant difference in CVD symptom knowledge and selfmanagement behavior according to the type of disease, type of medication taken, and risk of stroke (Table 1).

The effects of CVD symptom knowledge on selfmanagement behavior

In the first step of the hierarchical regression analysis, age, education, and duration of illness, which were significantly affected by self-management behavior, were included in the model as independent variables (Adj $\mathrm{R} 2=.10, \mathrm{~F}=5.42, \mathrm{p}<.001$ ). In the second step CVD symptom knowledge increased by $28.3 \%$ ( $\beta$ $\left.=0.53, \Delta \mathrm{R}^{2}=.28, \mathrm{p}<.001\right)$. In the final model, age $(\beta$ $=-.14, \mathrm{p}=.041)$ and CVD symptom knowledge $(\beta=$ $.53, \mathrm{p}=<001)$ were identified as significant influencing factors. The total explanatory power of the final model was $38 \%($ Adj $\mathrm{R} 2=.38, \mathrm{~F}=17.21, \mathrm{p}<.001)$.

Table 1 Differences of CVD Symptom Knowledge and Self-management behaviors by Subjects' Characteristics $(N=120)$

\begin{tabular}{|c|c|c|c|c|c|c|}
\hline \multicolumn{2}{|c|}{ Variables Categories } & \multirow{3}{*}{$\begin{array}{c}\text { n (\%) or } \\
\mathbf{M} \pm \mathbf{S D} \\
69.3 \pm 11.5\end{array}$} & \multicolumn{2}{|c|}{ CVD symptom knowledge } & \multicolumn{2}{|c|}{$\begin{array}{c}\text { Self-management } \\
\text { behaviors }\end{array}$} \\
\hline & & & $\mathbf{M} \pm \mathbf{S D}$ & t or $F(p)$ & $\mathbf{M} \pm \mathbf{S D}$ & $\operatorname{tor} F(p)$ \\
\hline \multirow{4}{*}{ Age (years) } & & & $6.30 \pm 5.12$ & \multirow{4}{*}{$\begin{array}{c}4.51(.012) \\
\mathrm{a}>\mathrm{c} \dagger\end{array}$} & $35.32 \pm 6.51$ & \multirow{4}{*}{$\begin{array}{c}6.49(.002) \\
a>c \dagger\end{array}$} \\
\hline & $<65 \mathrm{a}$ & $40(33.3)$ & $8.08 \pm 4.77$ & & $37.40 \pm 6.19$ & \\
\hline & $65 \sim 74 \mathrm{~b}$ & $35(29.2)$ & $6.17 \pm 5.31$ & & $35.97 \pm 8.71$ & \\
\hline & $\geq 75 \mathrm{c}$ & $45(37.5)$ & $4.82 \pm 4.87$ & & $33.10 \pm 7.68$ & \\
\hline \multirow{2}{*}{ Gender } & Men & $71(59.2)$ & $6.49 \pm 4.78$ & \multirow{2}{*}{$0.25(.621)$} & $35.31 \pm 7.40$ & \multirow{2}{*}{$0.02(.893$} \\
\hline & Women & $49(40.8)$ & $6.02 \pm 5.61$ & & $35.45 \pm 8.67$ & \\
\hline
\end{tabular}


Cont... Table 1 Differences of CVD Symptom Knowledge and Self-management behaviors by Subjects' Characteristics $(N=120)$

\begin{tabular}{|c|c|c|c|c|c|c|}
\hline \multirow{4}{*}{ Education } & $\leq$ Elementary schoola & $51(42.5)$ & $5.08 \pm 5.12$ & \multirow{4}{*}{$\begin{array}{c}6.88(<.001) \\
\mathrm{c}>\mathrm{a}, \mathrm{b} \dagger \\
\mathrm{d}>\mathrm{b} \dagger\end{array}$} & $33.13 \pm 7.61$ & \multirow{4}{*}{$\begin{array}{c}6.29(.001) \\
\mathrm{c}>\mathrm{a} \dagger\end{array}$} \\
\hline & Middle schoolb & $24(20.0)$ & $4.21 \pm 4.55$ & & $34.85 \pm 8.42$ & \\
\hline & High schoolc & $23(19.2)$ & $9.22 \pm 3.88$ & & $38.82 \pm 7.22$ & \\
\hline & $\geq$ Colleged & $22(18.3)$ & $8.36 \pm 5.01$ & & $37.18 \pm 5.91$ & \\
\hline \multirow{2}{*}{ Living with } & Spouse or Children & $102(85.0)$ & $6.09 \pm 4.87$ & \multirow{2}{*}{$1.16(.282)$} & $35.35 \pm 8.08$ & \multirow{2}{*}{$0.09(, 926)$} \\
\hline & Alone & $18(15.0)$ & $7.50 \pm 6.34$ & & $35.81 \pm 7.92$ & \\
\hline \multirow{2}{*}{$\begin{array}{c}\text { Monthly } \\
\text { income } \\
\text { (10,000Won })\end{array}$} & $<200$ & $82(68.3)$ & $6.39 \pm 5.44$ & \multirow{2}{*}{$0.08(.778)$} & $35.16 \pm 8.41$ & \multirow{2}{*}{$0.31(.578)$} \\
\hline & $\geq 200$ & $38(31.7)$ & $6.11 \pm 4.40$ & & $3.5 .81 \pm 6.75$ & \\
\hline \multirow{2}{*}{$\begin{array}{l}\text { Alcohol } \\
\text { drinking }\end{array}$} & Yes & $37(30.8)$ & $5.87 \pm 5.11$ & \multirow{2}{*}{$1.94(.166)$} & $36.36 \pm 7.43$ & \multirow{2}{*}{$1.15(.220)$} \\
\hline & No & $83(69.2)$ & $7.27 \pm 5.06$ & & $34.93 \pm 8.09$ & \\
\hline \multirow{2}{*}{ Smoking } & Yes & $18(15.0)$ & $8.28 \pm 5.14$ & \multirow{2}{*}{$3.22(.075)$} & $33.92 \pm 4.83$ & \multirow{2}{*}{$1.29(.258)$} \\
\hline & No & $102(85.0)$ & $5.95 \pm 5.06$ & & $35.62 \pm 8.30$ & \\
\hline \multirow{4}{*}{$\begin{array}{c}\text { Time since } \\
\text { diagnosed } \\
\text { (month) }\end{array}$} & & $46.2 \pm 39.1$ & $6.30 \pm 4.77$ & \multirow{4}{*}{$0.21(.808)$} & $35.36 \pm 7.91$ & \multirow{4}{*}{$\begin{array}{c}3.97(.021) \\
\mathrm{b}<\mathrm{c} \uparrow\end{array}$} \\
\hline & $\leq 12 \mathrm{a}$ & $34(28.4)$ & $6.50 \pm 5.40$ & & $33.31 \pm 8.06$ & \\
\hline & $13 \square 35 \mathrm{~b}$ & $19(15.8)$ & $6.84 \pm 5.96$ & & $37.76 \pm 8.11$ & \\
\hline & $\geq 36 \mathrm{c}$ & $67(55.8)$ & $6.04 \pm 4.77$ & & $35.73 \pm 7.42$ & \\
\hline \multirow{6}{*}{ Comorbidity } & Yest & $97(80.3)$ & $6.35 \pm 4.77$ & \multirow{6}{*}{$1.06(.306)$} & $34.67 \pm 7.73$ & \multirow{6}{*}{$1.38(.255)$} \\
\hline & Hypertension & $68(56.7)$ & $6.09 \pm 5.06$ & & $35.73 \pm 7.78$ & \\
\hline & Diabetes mellitus & $31(25.8)$ & $7.10 \pm 4.93$ & & $34.67 \pm 7.24$ & \\
\hline & $\begin{array}{c}\text { Peripheral vascular } \\
\text { disease }\end{array}$ & $21(17.5)$ & $5.90 \pm 5.42$ & & $35.27 \pm 8.42$ & \\
\hline & Heart failure & $19(15.8)$ & $6.52 \pm 5.13$ & & $34.76 \pm 7.30$ & \\
\hline & Previous stroke & $17(14.2)$ & $6.13 \pm 4.53$ & & $33.81 \pm 7.92$ & \\
\hline \multirow{4}{*}{ Medication } & None & $5(4.2)$ & $3.60 \pm 2.88$ & \multirow{4}{*}{$1.028(.392)$} & $34.17 \pm 5.05$ & \multirow{4}{*}{$.270(.847)$} \\
\hline & Antiplatelets & $30(25.0)$ & $6.90 \pm 4.95$ & & $35.26 \pm 7.91$ & \\
\hline & NOAC & $45(37.5)$ & $6.82 \pm 5.40$ & & $35.94 \pm 8.68$ & \\
\hline & Warfarin & $40(33.3)$ & $5.60 \pm 5.12$ & & $34.97 \pm 7.90$ & \\
\hline \multirow{3}{*}{$\begin{array}{c}\mathrm{CHA}_{2} \mathrm{DS}_{2} \\
\text {-VASc } \\
\text { score }\end{array}$} & Low risk (0) & $9(7.5)$ & $7.71 \pm 4.36$ & \multirow{3}{*}{$1.05(.353)$} & $35.87 \pm 5.45$ & \multirow{3}{*}{$1.38(.255)$} \\
\hline & Intermediate risk (1) & $12(10.0)$ & $7.27 \pm 4.50$ & & $37.59 \pm 6.53$ & \\
\hline & High risk $(>2)$ & $99(82.5)$ & $5.92 \pm 5.30$ & & $34.93 \pm 8.6$ & \\
\hline
\end{tabular}


†Scheffé test; ${ }^{\mathrm{N}}$ Multiple responses; CVD, cardiocerebrovascular disease; NOAC, new oral anti-coagulants; $\mathrm{CHA}_{2} \mathrm{DS}-\mathrm{VASc}$ score, congestive heart failure, hypertension, age, diabetes mellitus, prior stroke-vascular disease, age, sex female scheme score

Table 2 Levels of CVD Symptom Knowledge $(N=120)$

\begin{tabular}{|c|c|c|c|c|}
\hline Variables & Categories (items) & Range & M \pm SD & Correct answer (\%) \\
\hline \multicolumn{2}{|c|}{ CVD symptom knowledge, Total (17) } & $0 \sim 17$ & $6.30 \pm 5.12$ & 37.0 \\
\hline \multirow{2}{*}{ Stroke (5) } & $0 \sim 5$ & $2.21 \pm 2.11$ & 44.0 \\
\cline { 2 - 5 } & Myocardial infarction (5) & $0 \sim 5$ & $1.71 \pm 1.54$ & 34.0 \\
\cline { 2 - 5 } & Heart failure (7) & $0 \sim 7$ & $2.28 \pm 2.15$ & 32.0 \\
\hline
\end{tabular}

CVD, cardio-cerebrovascular disease

Table 3 Influence of CVD Symptom Knowledge on Self-management behaviors adjusted for Covariates by Hierachical Regression Analysis $(N=120)$

\begin{tabular}{|c|c|c|c|c|c|c|c|c|c|c|}
\hline \multirow{2}{*}{ Variables } & \multicolumn{5}{|c|}{ Model 1} & \multicolumn{5}{|c|}{ Model 2} \\
\hline & B & SE & $\beta$ & $\mathbf{t}$ & $\mathbf{p}$ & B & SE & $\beta$ & $\mathbf{t}$ & $\mathbf{p}$ \\
\hline (Constant) & 51.15 & 8.54 & & 6.49 & $<.001$ & 42.97 & 3.74 & & 6.33 & $<.001$ \\
\hline Age (years) & -1.12 & 0.90 & -.17 & -1.62 & .026 & -0.85 & 0.52 & -.14 & -1.70 & .041 \\
\hline Education & 1.40 & 0.83 & .83 & 1.68 & .095 & 0.74 & 0.45 & .10 & 1.04 & .300 \\
\hline Time since diagnosed(Month) & 0.01 & 0.02 & .04 & 0.46 & .644 & 0.01 & 0.01 & .15 & 0.89 & .377 \\
\hline \multirow[t]{2}{*}{ CVD symptom knowledge } & & & & & & 0.85 & 0.12 & .53 & 6.80 & $<.001$ \\
\hline & \multicolumn{5}{|c|}{$\begin{array}{c}\text { Adj. } \mathrm{R}^{2}=.10 \\
\mathrm{~F}=5.42, \mathrm{p}<.001\end{array}$} & \multicolumn{5}{|c|}{$\begin{array}{c}\text { Adj. } \mathrm{R}^{2}=.38, \Delta \mathrm{R}^{2}=.28 \\
\mathrm{~F}=17.21, \mathrm{p}<.001\end{array}$} \\
\hline
\end{tabular}

CVD, cardio-cerebrovascular disease

\section{Discussion}

The purpose of this study was to provide basic data for nursing interventions targeted at patients with $\mathrm{AF}$, through the assessment of stroke risk, CVD symptom knowledge, and self-management behavior. The average age of the participants was 69.3 years, and $82.5 \%$ of the high-risk group had experienced stroke. In a previous study, the mean age of patients with AF was 74 years, and $87.7 \%$ were in the high-risk group ${ }^{[14]}$. One possible reason for the difference in the percentage of high-risk patients between the present study and the previous study may be that the average age of the participants in the present study was lower than that of the previous study. 
As indicated by the scoring system of the $\mathrm{CHA}_{2} \mathrm{DS}_{2}$ VASc score, the risk of stroke increases with age. The risk factors found in a study by Tze-fan ${ }^{[15]}$ differed from those in the present study in complications age, hypertension, being female, and heart failure. AF has been found to be 2.7 times higher in patients with hypertension, 1.6 times higher in patients with heart failure, and 1.3 times higher in patients with diabetes, according to the associated risk of stroke ${ }^{[16]}$. Comorbidities should be managed together to reduce the risk of cardio- cerebrovascular disease. However, in patients with coronary artery disease, accompanying diseases such as hyperlipidemia and hypertension have not been recognized as risk factors ${ }^{[17]}$. Since patients with AF are expected to have similar risk factors, it is necessary to emphasize the need for selfmanagement of comorbid diseases to reduce the risk of stroke. The average score for CVD symptom knowledge was $6.30 \pm 5.12$ points, out of 17 possible points, in the present study, with a correct answer rate of $37 \%$. It was much lower than the previous study ${ }^{[12]}$, which reported a correct answer rate of $62 \%$ with using the same measurement tool. Emphasis on the prevent stroke ${ }^{[18]}$, early education of AF patients, and appropriate coping measures should be promptly provided. In particular, for knowledge about heart failure symptoms, the average rate of correct responses was $32 \%$, which was lower compared with stroke and myocardial infarction symptoms. In order to raise awareness of CVD, it is necessary to plan interventions that focus on prevention of disease progression and exacerbation, and health promotion. The average score for self-management behavior in the present study was $35.32 \pm 6.51$, which was lower than that of a previous study ${ }^{[13]}$. that used the same measurement tool. The average score in that study was 38.6. Lack of information on diseases and complications is likely to be an obstacle to effective selfmanagement behavior.

In the present study, there were significant differences in CVD symptom knowledge by age and education level. In a study of patients with AF, conducted by the American Arrhythmia Association knowledge about stroke symptoms was higher with age and more education, similar to the results of the present study ${ }^{[19]}$. Education should be provided to groups of patients with $\mathrm{AF}$ that are at higher risk for stroke, and levels of $\mathrm{AF}$ management should be assessed to improve patient knowledge about acute symptoms of stroke and coping with symptoms.

In this study, hierarchical regression analysis was performed to determine the effect of symptom knowledge on self-management behavior when controlling the effects of general and disease-related covariates. In the univariate analysis, age, education level, and duration of illness were the significant influencing factors for age, and the explanatory power was $10 \%$. Symptom knowledge was increased to $38 \%$. In other words, symptom knowledge was a significant factor that increased $28 \%$ of self-management behavior. These findings support those of a previous study that indicated that diseaserelated knowledge is a highly influencing factor of selfmanagement behavior in patients with heart failure ${ }^{[20]}$. In older patients, poorer self-management behavior is expected due to decreased understanding of the disease, slower awareness of the disease, and more difficulty in changing lifestyle behaviors. Therefore, before nursing intervention, personalized strategies should be provided after evaluating the age and learning competencies of the patient. One previous study found that patients with AF had a high level of uncertainty and depression that could affect self-management behavior ${ }^{[9]}$. In the future, it is necessary to conduct repeated studies to identify the influencing factors of self-management behaviors, including symptom knowledge and psychosocial variables, in patients with $\mathrm{AF}$.

This study had two major limitations. First, results from the sample of outpatients may not be generalizable to all patients with AF. Second, this was a crosssectional study that used self-reported data, and the causal relationships among stroke risk, knowledge, and self-management behavior could not be inferred. However, this study is meaningful in that it demonstrates that in patients with $\mathrm{AF}$, knowledge about symptoms related to complications is insufficient for effective selfmanagement.

\section{Conclusions}

In this study, $82.5 \%$ of patients with $\mathrm{AF}$ were at high risk for stroke. The rate of correct CVD symptom knowledge was $37 \%$. The level of self-management 
behavior for AF management was $35.32 \pm 6.51$, which was not high. Results of the hierarchical regression analysis indicated that CVD symptom knowledge was an independent factor of self-management behavior, and explanation ability was $38 \%$. In order to improve self-management behavior in patients with $\mathrm{AF}$, it is necessary to provide education that focuses on warning symptoms of complications such as stroke, myocardial infarction, and heart failure and is tailored to the age and knowledge level of the patient.

\section{References}

1. January CT, Wann LS, Alpert JS, Calkins H, Cigarroa JE, Cleveland JC, et al. 2014 AHA/ ACC/HRS Guideline for the management of patients with atrial fibrillation: a report of the American College of Cardiology/American Heart Association task force on practice guidelines and the heart rhythm society. Journal of the American College of Cardiology. 2014;64(21):e1-e76. doi. org/10.1016/j.jacc.2014.03.022

2. Eun JN, Cho JG, Kim SS, Park HW, Lee KH, Yoon NS, et al. Prevalence of atrial fibrillation in the elderly in Korea. Journal of the Korean Geriatrics Society. 2016;20(1):29-35. doi.org/10.4235/ jkgs.2016.20.1.29

3. Kirchhof P, Benussi S, Kotecha D, Ahlsson A, Atar D, Casadei B, et al. 2016 ESC Guidelines for the management of atrial fibrillation developed in collaboration with EACTS. European Heart Journal. 2016;37(38):2893-962.doi.org/10.1016/j. crvasa.2016.11.005

4. Kim BJ, Han MK, Park TH, Park SS, Lee KB, Lee $\mathrm{BC}$, et al. Current status of acute stroke management in Korea: a report on a multicenter, comprehensive acute stroke registry. International Journal of Stroke. 2014;9(4):514-8.doi.org/10.1111/ijs.12199

5. Tu HT, Campbell BC, Churilov L, Kalman JM, Lees KR, Lyden PD, et al. Frequent early cardiac complications contribute to worse stroke outcome in atrial fibrillation. Cerebrovascular Diseases. 2011;32(5):454-60.doi.org/10.1159/000332028

6. Diouf I, Magliano DJ, Carrington MJ, Stewart S, Shaw JE. Prevalence, incidence, risk factors and treatment of atrial fibrillation in Australia: the Australian diabetes, obesity and lifestyle(AUsDiab) longitudinal, population cohort study. International Journal of Cardiology. 2016;205:127-32. doi. org/10.1016/j.ijcard.2015.12.013

7. Fournaise, A., Skov, J., Bladbjerg, E. M., Leppin, AStroke risk perception in atrial fibrillation patients is not associated with clinical stroke risk. Journal of Stroke and Cerebrovascular Diseases. 2015; 24(11), 2527-2532.

8. Tai YJ, Yan B. Minimising time to treatment: targeted strategies to minimise time to thrombolysis for acute ischaemic stroke. Internal Medicine Journal. 2013;43(11):1176-82.doi.org/10.1111/imj.12204

9. McCabe PJ, Schad S, Hampton A, Holland DE. Knowledge and self-management behaviors of patients with recently detected atrial fibrillation. Heart \& Lung: The Journal of Acute and Critical Care. 2008;37(2):79-90.doi.org/10.1016/j. hrtlng.2007.02.006

10. Noh JH, Shin YH. Probability of stroke, knowledge of stroke, and health-promoting lifestyle in stroke risk groups. Journal of the Korean Academy of Fundamentals of Nursing. 2014;21(2):174-82.doi. org/10.7739/jkafn.2014.21.2.174

11. Lip GY. The $\mathrm{CHA}_{2} \mathrm{DS}_{2}$-VASc score for stroke risk stratification in patients with atrial fibrillation: a brief history. European Heart Journal. 2015;36(42):2880-5.doi.org/10.1093/eurheartj/ ehv431

12. Choi SK, Kim IS. Effects of a cardiocerebrovascular disease prevention education program for postmenopausal middle-aged women. Journal of Korean Academy of Nursing. 2015;45(1):25-34. doi.org/10.4040/jkan.2015.45.1.25

13. Xu, W., Sun, G., Lin, Z., Chen, M., Yang, B., Chen, H. et al. Knowledge, attitude, and behavior in patients with atrial fibrillation undergoing radiofrequency catheter ablation. Journal of interventional cardiac electrophysiology, 2010, 28(3), 199-207.

14. Gallagher AM, Setakis E, Plumb JM, Clemens A, van Staa T-P. Risks of stroke and mortality associated with suboptimal anticoagulation in atrial fibrillation patients. Thrombosis and Haemostasis. 2011;106(5):968-77.

15. Tze-Fan C. Validation of a modified CHA(2)DS(2)VASc score for stroke risk stratification in asian patients with atrial fibrillation: a nationwide cohort study. Stroke. 2016;47:2462-69.doi.org/10.1161/ STROKEAHA.116.013880

16. Lin LY, Lee CH, Yu CC, Tsai CT, Lai LP, Hwang JJ, 
et al. Risk factors and incidence of ischemic stroke in Taiwanese with nonvalvular atrial fibrillation-a nation wide database analysis. Atherosclerosis. 2011;217(1):292-5.

17. Yang IS, Choi DH, Kang YH. The awareness of cardiovascular risk factors and its correlates in patients with coronary artery diseases. Korean Journal of Adult Nursing. 2010;22(5):499-508.

18. Han EJ, Kim JS. Effects of symptom recognition and health behavior compliance on hospital arrival time in patients with acute myocardial infarction.
Korean Journal of Adult Nursing. 2015;27(1):8393. doi.org/10.7475/kjan.2015.27.1.83

19. Xu W, Sun G, Lin Z, Chen M, Yang B, Chen H, et al. Knowledge, attitude, and behavior in patients with atrial fibrillation undergoing radiofrequency catheter ablation. Journal of Interventional Cardiac Electrophysiology. 2010;28(3):199-207.

20. Son YJ, Kim SH, Kim GY. Factors influencing adherence to self care in patients with chronic heart failure. Korean Journal of Adult Nursing. 2011;23(3):244-54. 